\title{
Enzyme Inhibition and In Silico Studies of New Synthetic N-Substituted- (4-Bromophenyl)-4-Ethoxybenzenesulfonamides
}

\author{
Naheed Riaz ${ }^{1 *}$, Muhammad Iftikhar ${ }^{1}$, Muhammad Saleem ${ }^{1}$, Shahnawaz ${ }^{1}$, Aziz ur Rehman ${ }^{2}$, Ishtiaq Ahmed ${ }^{3}$, \\ Safdar Hussain¹, Muhammad Ashraf ${ }^{1 *}$, Zahid Nawaz ${ }^{1}$, Jameel Rahman ${ }^{1}$ and Mariya al Rashida ${ }^{4}$ \\ ${ }^{1}$ Department of Chemistry, The Islamia University of Bahawalpur, Pakistan \\ ${ }^{2}$ Department of Chemistry, Government College University, Lahore, Pakistan \\ ${ }^{3}$ Karlsruhe Institute of Technology (KIT), Institute for Biological Interfaces (IBG-1), Hermann-von-Helmholtz-Platz, Karlsruhe, Germany \\ ${ }^{4}$ Department of Chemistry, Forman Christian College (A Chartered University), Lahore, Pakistan
}

Submission: September 08, 2020; Published: December 01, 2020

"Corresponding author: Naheed Riaz and Muhammad Ashraf, Department of Chemistry, The Islamia University of Bahawalpur 63100, Bahawalpur, Pakistan

Abstract

A series of new N-substituted-(4-bromophenyl)-4-ethoxybenzenesulfonamides (5a-o) were synthesized and evaluated for enzyme inhibition potential. The task was accomplished by the reaction of 4-bromobenzenesulfonyl chloride

a. with 4-ethoxyaniline

b. to get the intermediate 4-bromophenyl-4-ethoxybenzenesulfonamide in the first step.

The compound 3 on further reaction with different electrophiles (4a-o) yielded the target compounds 5a-o, which were characterized with the help of FTIR, ${ }^{1} \mathrm{H}-,{ }^{13} \mathrm{C}$-NMR spectroscopic and EI-MS \& HR-EI-MS spectrometric data. These sulfonamides (5a-o) were evaluated for their acetylcholinesterase (AChE) and $\alpha$-glucosidase inhibitory potential. Compounds $5 \mathrm{l}, 5 \mathrm{n}, 5 \mathrm{~g}, 5 \mathrm{j}$ and $5 \mathrm{~h}$ exhibited excellent potential against AChE with IC ${ }_{50}$ values of $52.63 \pm 0.14,82.75 \pm 0.16,92.13 \pm 0.15,92.52 \pm 0.16$ and $98.72 \pm 0.12 \mu \mathrm{M}$, respectively. Compounds $5 \mathrm{~h}, 5 \mathrm{j}, 5 \mathrm{c}, 5 \mathrm{~d}$ and $5 \mathrm{l}$ were found potent inhibitors of $\alpha$-glucosidase with $\mathrm{IC}_{50}$ values of $57.38 \pm 0.19,123.36 \pm 0.19,123.42 \pm 0.19,124.35 \pm 0.15$ and $124.74 \pm 0.18 \mu \mathrm{M}$, respectively. The activity results were also substantiated by in silico studies.

Keywords: Sulfonamide; Enzyme inhibition; Acetylcholinesterase inhibition; $\alpha$-Glucosidase inhibition; Molecular docking studies

\section{Introduction}

The sulfonamides (-SO $\left.\mathrm{SO}_{2} \mathrm{NH}-\right)$ are proven as fascinating compounds as a main core for different bioactivities and can be more like a string of distinguished pearls. Sulfonamides are called sulfa drugs, which were the first antibacterial agents to be used systemically and paved the way for the antibiotic revolution in medicine. The first medicine of this class was Prontosil discovered as effective treatment of a range of bacterial infections. It had strong protective action against infections caused by Streptococci, including blood infections, childbed fever, and erysipelas [1]. Sulfonamides are structurally like p-aminobenzoic acid (PABA), a cofactor that is needed by bacteria for the synthesis of folic acid. Various medicines for the treatment of different diseases having the $-\mathrm{SO}_{2} \mathrm{NH}$ - function are available in the market like sulfafurazole as children antibiotic, gliquidone as an antidiabetic for diabetes mellitus type-2, furosemide as a diuretic, zonisamide to treat epilepsy and Parkinson, mafenide as an antibiotic to treat skin infections, and dasabuvir to kill hepatitis C virus (Figure 1).

Clinically, sulfonamides are used to treat several urinary tract and gastrointestinal infections [2]. This group of compounds acts as antitumor agents by inhibiting the carbonic anhydrase. 
Sulfonamides antibiotics inhibit the conversion of PABA into folic acid and thus ultimately inhibit the synthesis of purines and DNA [3,4]. Besides, antibacterial properties, sulfonamides also have other activities, like carbonic anhydrase inhibitors [5,6], anticancer, anti-inflammatory and analgesic agents [7], 63adrenergic receptor agonists [8], PC-1 inhibitors [9], antifungal
[10] and antiviral agents [11]. Keeping in view the broadspectrum biological activities of sulfonamides, we designed a straightforward and efficient method to synthesize high yield sulfonamides and believe that this route will further be used for the synthesis of biologically active compounds.

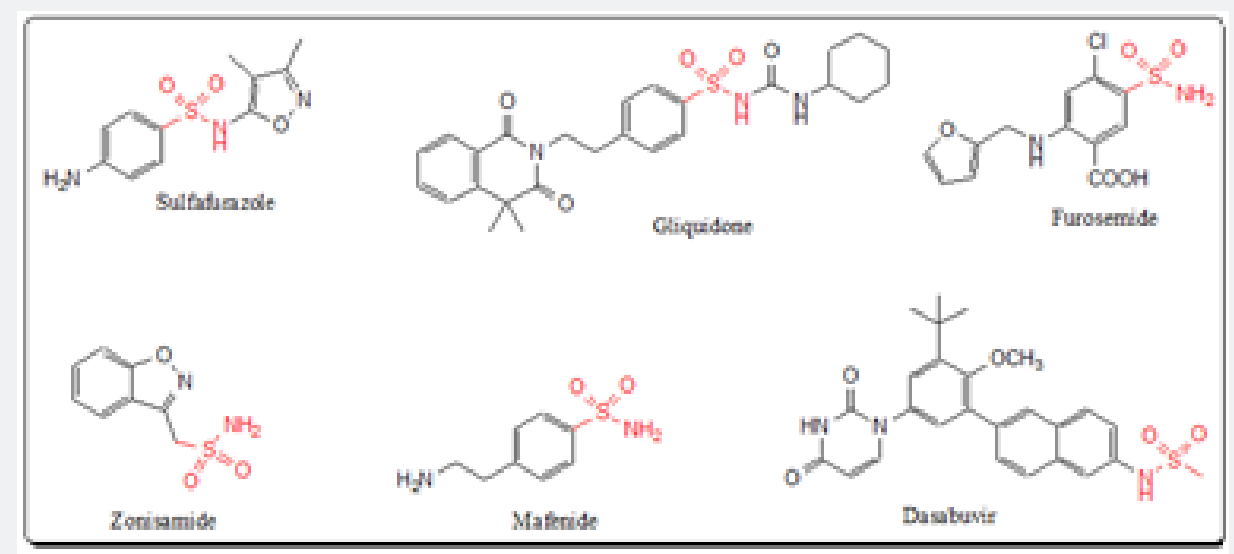

Figure 1: Some available drugs containing sulfonamide functionality in their structures.

Acetylcholinesterase (AChE, EC 3.1.1.7) is an enzyme that catalyzes the breakdown of acetylcholine esters that function as neurotransmitters. AChE is found mainly at neuromuscular junctions and in chemical synapses of the cholinergic type, where its activity serves to terminate synaptic transmission. It belongs to carboxylesterase family of enzymes that are primary target of inhibition by organophosphorus compounds such as nerve agents and pesticides [12], $\alpha$-Glucosidase (EC, 3.2.1.20) inhibitors are used for the treatment of diabetes mellitus type- 2 by inhibiting the digestion of carbohydrates. Conversely, carbohydrates are not converted into simple sugars by the enzyme present on cells lining the intestine. Hence, immediate post-prandial increase is restricted and sudden rise in blood sugar levels does not occur [13].

Structure-based drug design for an enzyme target has been facilitated with crystal structures which enable the computational searches to identify 'lead' compounds for refinement. There are feasible large-scale computational approaches which include analysis of off-target activity and combining suitable pharmacophores for enzyme combinations. However, such compounds still must be synthesized and tested experimentally to confirm the predicted inhibitory effects against each of the targets [14]. The aim of the present study was to synthesize alkyl/aralkyl substituted-N-(4-ethoxyphenyl)-4-bromobenzenesulfonamides (5a-o) and investigate them for their enzyme inhibitory activities against AChE and $\alpha$-glucosidase in search for the 'lead' compounds against these enzymes of therapeutical importance. Synthesis of the intermediate and target compounds were carried out according to the protocol as shown in Figure 2.

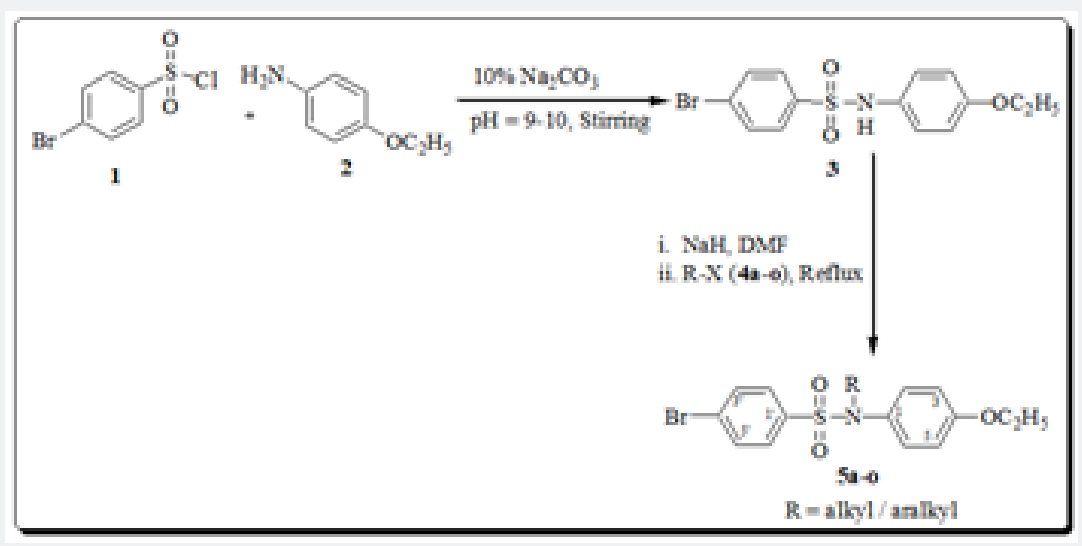

Figure 2: Protocol for the synthesis of compounds 5a-o. 
Compound 5a was synthesized as off-white amorphous solid. The molecular formula was found to be $\mathrm{C}_{16} \mathrm{H}_{18} \mathrm{BrNO}_{3} \mathrm{~S}$ since HR-EIMS showed molecular ion [M] ${ }^{+}$peak at $m / z 383.0199$ (calculated for $\left.\mathrm{C}_{16} \mathrm{H}_{18} \mathrm{BrNO}_{3} \mathrm{~S} ; 383.0190\right)$. The IR spectrum exhibited the presence of aromatic and aliphatic hydrogens $\left(3039,2968 \mathrm{~cm}^{-1}\right)$, unsaturation (1625-1576 $\left.\mathrm{cm}^{-1}\right)$, sulfonyl $\left(1341 \mathrm{~cm}^{-1}\right)$ and ethoxy $\left(1258 \mathrm{~cm}^{-1}\right)$ moieties in the molecule. The aliphatic region of the ${ }^{1} \mathrm{H}$-NMR spectrum of 5 a showed signals for $N$-ethyl and $O$-ethyl moieties at $\delta 1.05\left(3 \mathrm{H}, \mathrm{t}, \mathrm{J}=7.0 \mathrm{~Hz}, \mathrm{H}-2^{\prime \prime}\right), 1.39(3 \mathrm{H}, \mathrm{t}, \mathrm{J}=7.0 \mathrm{~Hz}$, $\left.\mathrm{CH}_{3}-\mathrm{CH}_{2}-\mathrm{O}\right), 3.53\left(2 \mathrm{H}, \mathrm{q}, \mathrm{J}=7.0 \mathrm{~Hz}, \mathrm{H}-1^{\prime \prime}\right)$ and $3.99(2 \mathrm{H}, \mathrm{q}, \mathrm{J}=7.0$ $\mathrm{Hz}, \mathrm{CH}_{3}-\mathrm{CH}_{2}-\mathrm{O}$ ). The aromatic region of the spectrum showed two pairs of ortho-coupled doublets at $\delta 6.79(2 \mathrm{H}, \mathrm{d}, \mathrm{J}=8.5 \mathrm{~Hz}$, $\mathrm{H}-2,6), 6.89(2 \mathrm{H}, \mathrm{d}, \mathrm{J}=8.5 \mathrm{~Hz}, \mathrm{H}-3,5), 7.44(2 \mathrm{H}, \mathrm{d}, \mathrm{J}=8.5 \mathrm{~Hz}$, $\left.\mathrm{H}-2^{\prime}, 6^{\prime}\right)$ and $7.56\left(2 \mathrm{H}, \mathrm{d}, \mathrm{J}=8.5 \mathrm{~Hz}, \mathrm{H}-3^{\prime}, 5^{\prime}\right)$, which were assigned to two p-substituted phenyl rings attached to the sulfonamide functionality.
The ${ }^{13} \mathrm{C}$-NMR both broad band (BB) and distortion less enhancement by polarization transfer (DEPT) spectra of compound 5a showed altogether twelve carbon signals for sixteen carbons corroborated the presence of two methyl, two methylene, four methine and four quaternary carbons. The presence of an ethoxy and nitrogen substituted ethyl group was confirmed due to signals at $\delta 15.8,63.7 \& 15.0,45.9$, respectively. The confirmation of two 1,4-disubstituted benzene rings was done due to the resonances at $\delta 130.6(\mathrm{C}-1), 129.2(\mathrm{C}-2,6), 115.8(\mathrm{C}-3,5), 158.6(\mathrm{C}-5) \& 137.6$ (C-1'), 130.1 (C-2',6'), $132.0\left(\mathrm{C}^{\prime} 3^{\prime}, 5^{\prime}\right), 127.5$ (C-5'), respectively. Based on this evidence, the structure of 5 a was established as 4-bromo-N-(4-ethoxyphenyl)-N-ethylbenzene sulfonamide. Other compounds, 5b-o, were also characterized by using IR, EI-MS, HREI-MS, ${ }^{1} \mathrm{H}-\mathrm{NMR}$, and ${ }^{13} \mathrm{C}-\mathrm{NMR}$. Their details are described in the experimental section.

Table 1: Alkyl/aralkyl substituted groups (5a-o).

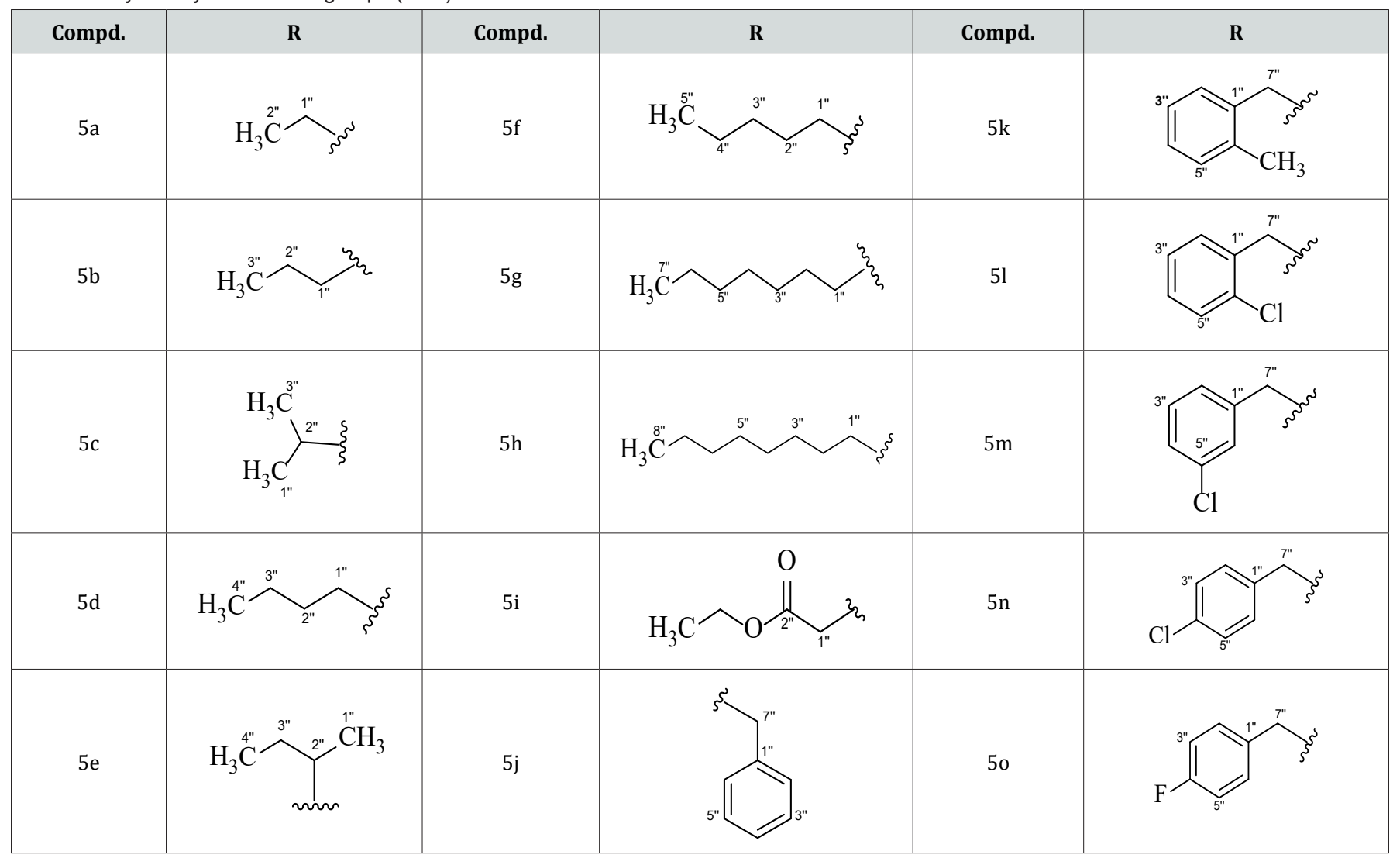

\section{Enzyme Inhibition Studies}

\section{AChE and $\alpha$-glucosidase inhibition activity}

The biological screening of these newly synthesized sulfonamides was carried out against AChE and $\alpha$-glucosidase enzymes. The results showed that these compounds exhibited good inhibitory potential against AChE and $\alpha$-glucosidase (Table
2). Amongst the tested compounds, 5l, $5 \mathrm{n}, 5 \mathrm{~g}, 5 \mathrm{j}, 5 \mathrm{~h}$ exhibited excellent AChE inhibition with $\mathrm{IC}_{50}$ values of $52.63 \pm 0.14,82.75 \pm$ $0.16,92.13 \pm 0.15,92.52 \pm 0.16$ and $98.72 \pm 0.12 \mu \mathrm{M}$, respectively. The compounds, $5 \mathrm{~h}, 5 \mathrm{j}, 5 \mathrm{c}, 5 \mathrm{~d}, 5 \mathrm{l}$ showed potent inhibitory activity against $\alpha$-glucosidase with $\mathrm{IC}_{50}$ values of $57.38 \pm 0.19$, $123.36 \pm 0.19,123.42 \pm 0.19,124.35 \pm 0.15$ and $124.74 \pm 0.18 \mu \mathrm{M}$, respectively (Table 2). 
Table 2: AChE and a-glucosidase inhibitory activities of compounds 5a-o.

\begin{tabular}{|c|c|c|c|c|}
\hline \multirow{2}{*}{ Comp. } & \multicolumn{2}{|c|}{ AChE } & \multicolumn{2}{|c|}{ Yeast $\alpha$-Glucosidase } \\
\hline & Inhibition (\%) at $0.5 \mathrm{mM}$ & $\mathrm{IC}_{50}(\mu \mathrm{M})$ & Inhibition (\%) at $0.5 \mathrm{mM}$ & $\mathrm{IC}_{50}(\mu \mathrm{M})$ \\
\hline $5 a$ & $21.54 \pm 0.14$ & - & $56.53 \pm 0.29$ & $447.24 \pm 0.18$ \\
\hline $5 b$ & $64.38 \pm 0.19$ & $416.54 \pm 0.16$ & $79.43 \pm 0.23$ & $273.78 \pm 0.16$ \\
\hline $5 c$ & $75.45 \pm 0.21$ & $365.32 \pm 0.15$ & $85.15 \pm 0.27$ & $123.42 \pm 0.19$ \\
\hline $5 d$ & $12.36 \pm 0.12$ & - & $85.23 \pm 0.28$ & $124.35 \pm 0.15$ \\
\hline $5 e$ & $78.67 \pm 0.21$ & $302.25 \pm 0.17$ & $81.75 \pm 0.28$ & $253.52 \pm 0.16$ \\
\hline $5 f$ & $86.23 \pm 0.18$ & $157.45 \pm 0.14$ & $51.63 \pm 0.26$ & $<500$ \\
\hline $5 g$ & $89.47 \pm 0.19$ & $92.13 \pm 0.15$ & $82.58 \pm 0.23$ & $153.54 \pm 0.17$ \\
\hline $5 \mathrm{~h}$ & $89.26 \pm 0.17$ & $98.72 \pm 0.12$ & $87.34 \pm 0.25$ & $57.38 \pm 0.19$ \\
\hline $5 \mathrm{i}$ & $37.53 \pm 0.15$ & - & $68.27 \pm 0.23$ & $312.42 \pm 0.18$ \\
\hline $5 j$ & $89.38 \pm 0.21$ & $92.52 \pm 0.16$ & $85.22 \pm 0.28$ & $123.36 \pm 0.19$ \\
\hline $5 \mathrm{k}$ & $89.75 \pm 0.23$ & $112.84 \pm 0.18$ & $84.83 \pm 0.25$ & $148.53 \pm 0.16$ \\
\hline 51 & $91.46 \pm 0.19$ & $52.63 \pm 0.14$ & $82.52 \pm 0.26$ & $124.74 \pm 0.18$ \\
\hline $5 \mathrm{~m}$ & $43.29 \pm 0.16$ & - & $23.41 \pm 0.23$ & - \\
\hline $5 \mathrm{n}$ & $90.54 \pm 0.21$ & $82.75 \pm 0.16$ & $84.39 \pm 0.27$ & $142.52 \pm 0.18$ \\
\hline 50 & $89.72 \pm 0.19$ & $142.63 \pm 0.15$ & $82.24 \pm 0.28$ & $172.38 \pm 0.19$ \\
\hline Eserine & $91.27 \pm 1.17$ & $0.04 \pm 0.001$ & - & - \\
\hline Acarbose & - & - & $65.73 \pm 1.93$ & $375.82 \pm 1.76$ \\
\hline
\end{tabular}

The sulfonamides with alkyl groups on nitrogen atom showed AChE inhibitory activity. Amongst these compounds, $5 \mathrm{~g}$ bearing n-heptyl and $5 \mathrm{~h}$ bearing n-octyl groups showed good inhibition against $\mathrm{AChE}$ with $\mathrm{IC}_{50}$ values of $92.13 \pm 0.15$ and $98.72 \pm 0.12 \mu \mathrm{M}$, respectively. However, it is found that there was a decrease in the activity with the decrease in carbon chain length on nitrogen atom, that is, increase in lipophilicity enhanced the activity. Amongst the benzyl substituted sulfonamides, compounds having o-chlorobenzyl substitution on the nitrogen atom pronounced the activity $\left(5 \mathrm{i}, \mathrm{IC}_{50} 52.63 \pm 0.14 \mu \mathrm{M}\right)$ whereas the compounds with p-chlorobenzyl ( $5 \mathrm{n}$ ) and only benzyl substitution (5j) on nitrogen atom were found to be the significant $\mathrm{AChE}$ inhibitors $\mathrm{IC}_{50}$ $82.75 \pm 0.16$ and $92.52 \pm 0.16 \mu \mathrm{M}$, respectively). The compound with m-chlorobenzyl substitution on nitrogen atom showed the least AChE inhibitory activity.

As for as the $\alpha$-glucosidase activity is concerned, the sulfonamides having n-octyl group (5h) attached to nitrogen offered potent inhibition $\left(\mathrm{IC}_{50} 57.38 \pm 0.19 \mu \mathrm{M}\right.$ ) whereas the activity decreased with the decrease in carbon chain length (Table 2) except the compound $5 \mathrm{~d}$ with n-butyl group on nitrogen (IC $_{50}$ $124.35 \pm 0.15 \mu \mathrm{M})$. These observations lead to the conclusion that increases in lipophilicity on nitrogen increased the anti- $\alpha$ glucosidase activity. Amongst the benzyl substituted sulfonamides, compounds having o-and p-chlorobenzyl substitution on nitrogen atom were found as good inhibitors of $\alpha$-glucosidase (IC ${ }_{50} 124.74$ $\pm 0.18 ; 142.52 \pm 0.18 \mu \mathrm{M}$, respectively). The isomer $5 \mathrm{~m}$ with m-chlorobenzyl group was the least inhibitor indicating that the substitution at m-position retarded the inhibition whereas the benzyl group without any substitution (5j) showed significant a-glucosidase inhibition ( IC $_{50} 123.36 \pm 0.19 \mu \mathrm{M}$ ).

\section{In silico Studies}

\section{AChE docking studies}

For docking studies against AChE enzyme, the most active compound 51 was selected. The crystal structure of hAChE (PDB id: $4 \mathrm{M} 0 \mathrm{E}, 2 \AA$ ) was downloaded from the PDB, the docking studies were performed according to our previously reported protocol [20] using Lead IT docking software [19]. Compound 5l was found to bind in the same region of the active site as that of cocrystallized inhibitor. Figure 3 shows docked conformation of 5l. The bromo phenyl ring was making a $\pi$-anion interaction with Asp74, a $\pi-\pi$ T-shaped interaction was observed between the same bromo phenyl ring with Tyr124 and Asp74. Another $\pi-\pi$ T-shaped interaction was seen between chloro phenyl ring and Phe338. A $\pi$-alkyl interaction was observed between the chloro group of same phenyl ring and Tyr337. Two more $\pi$-alkyl interactions were observed for the ethoxy side chain with Val294 and Tyr341. A $\pi-\pi$ stacked interaction was observed between the phenyl ring containing ethoxy substituent and Tyr341. Hydrogen bond was predicted between one of the sulfonamide oxygen atoms with Ty341. Another hydrogen bond was observed with the oxygen atom of the ethoxy group and Phe295. 


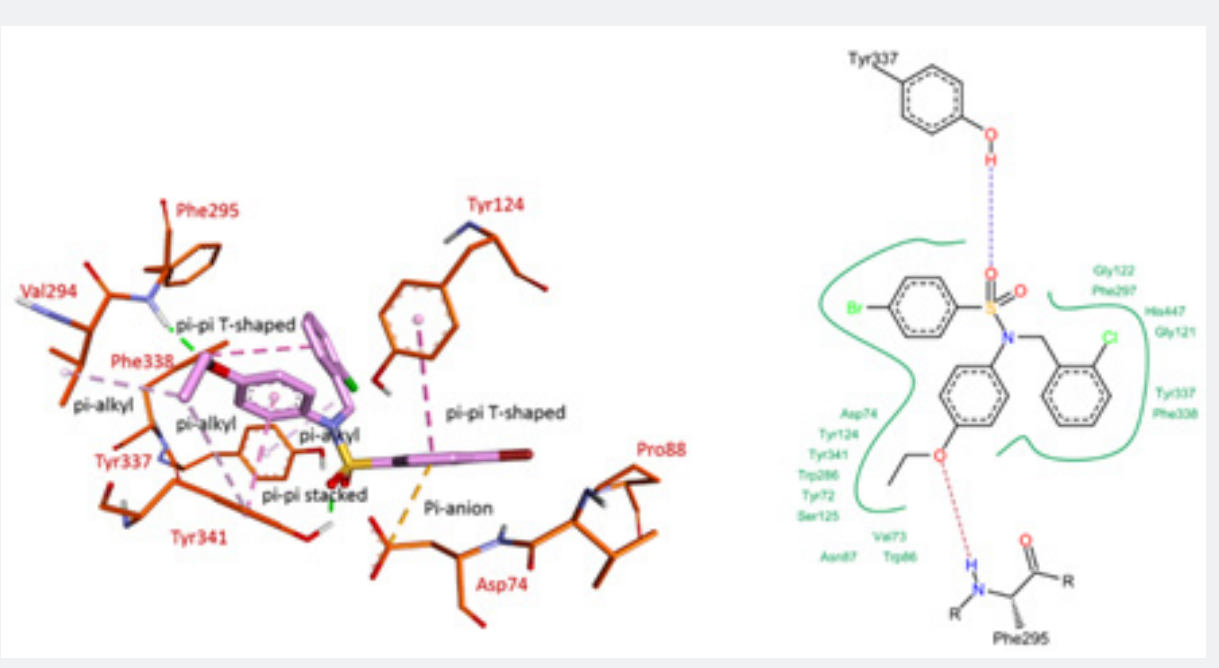

Figure 3: Binding site interactions of $5 \mathrm{I}$ inside active site of hAChE.

\section{$\alpha$-Glucosidase docking studies}

To get a better understanding of binding site interactions of the most active $\alpha$-glucosidase inhibitor, compound $5 \mathrm{~h}$ was docked into homology-built model of yeast $\alpha$-glucosidase according to our previously reported protocol [15]. The compound was found to bind in the active site of the enzyme. Several bonded and nonbonded interactions were observed that were responsible for the inhibitory activity of the compound. The $\mathrm{N}$-alkyl side chain was making $\pi$-alkyl interactions with amino acids Ala278, Leu218 and His245. Similarly, the ethoxy side chain was also making $\pi$-alkyl interactions with Phe177 and Tyr71. The bromo phenyl ring was making $\pi$-alkyl interactions with Arg439 and Arg312, it was also making a $\pi$-anion interaction with Asp408. The oxygen atom of ethoxy side chain was making hydrogen bond with Arg439 (Figure 4).

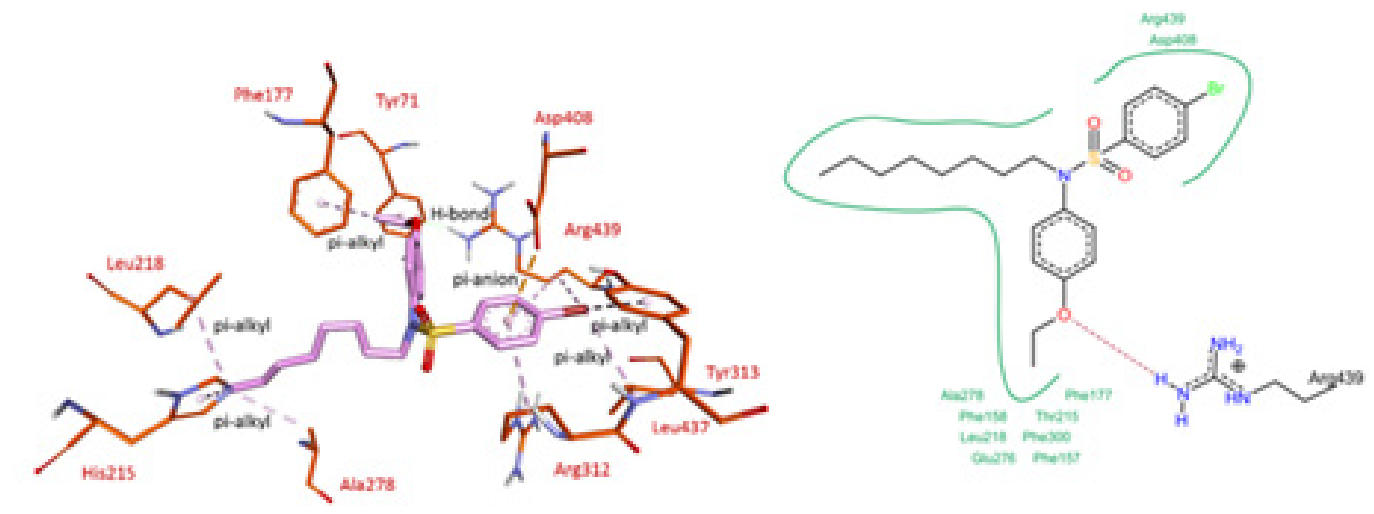

Figure 4: Binding site interactions of $5 \mathrm{~h}$ inside active site of $\alpha$-glucosidase.

\section{ADME properties of compounds (5a-o)}

The ADME (absorption, distribution, metabolism, excretion) are pharmacological properties of molecules were calculated by Med Chem Designer and data is given in Table 3. Permeability and solubility of the drugs are the two basic requirements for a drug to have good pharmacokinetic properties. In Lipinski's rules, molecular weights of compounds, log $\mathrm{P}$, number of hydrogen bond acceptors and hydrogen bond donors are associated with the permeability and solubility properties of molecules. The determination of polar surface area and molecular flexibility is associated with the oral bioavailability of drugs [18].

From the table it is observed that the compounds having higher $\log \mathrm{D}$ and $\log \mathrm{P}$ values and lower number of hydrogen bonds predict higher bioavailability of drugs. S+logP and MlogP are octanol-water distribution coefficients and molecules with values below 5 predict them to have drug-like properties in silico. 
TPSA is the topological polar surface area. A molecule with TPSA value of exceeding $140 \AA^{2}$, predicts the decreased bioavailability of molecule. In the present studies, all molecules showed excellent
TPSA values between 46.61 and $72.91 \AA^{2}$. Thus, all these molecules possess desirable drug-like TPSA property.

Table 3: ADME properties of compounds 5a-j.

\begin{tabular}{|c|c|c|c|c|c|c|c|}
\hline Comp & MlogP & S+logP & S+logD & MW & MNO & TPSA & HBDH \\
\hline $5 \mathrm{a}$ & 3.465 & 4.343 & 4.343 & 384.299 & 4 & 4 & 46.61 \\
\hline $5 \mathrm{~b}$ & 3.7 & 4.62 & 4.62 & 398.326 & 4 & 46.61 & 0 \\
\hline $5 \mathrm{c}$ & 3.7 & 4.705 & 4.705 & 398.326 & 46.61 & 0 \\
\hline $5 \mathrm{~d}$ & 3.93 & 5.056 & 5.056 & 412.353 & 46.61 & 0 \\
\hline $5 \mathrm{e}$ & 3.93 & 4.927 & 4.927 & 412.353 & 4 & 46.61 & 0 \\
\hline $5 \mathrm{f}$ & 4.155 & 5.486 & 5.486 & 426.38 & 4 & 46.61 & 0 \\
\hline $5 \mathrm{~g}$ & 4.592 & 6.486 & 6.486 & 454.434 & 4 & 46.61 & 0 \\
\hline $5 \mathrm{~h}$ & 4.805 & 6.982 & 6.982 & 468.461 & 4 & 46.61 & 0 \\
\hline $5 \mathrm{i}$ & 2.7 & 3.871 & 3.871 & 442.336 & 4 & 72.91 & 0 \\
\hline $5 \mathrm{j}$ & 4.37 & 5.2 & 5.2 & 446.37 & 4 & 46.61 & 0 \\
\hline $5 \mathrm{k}$ & 4.583 & 5.686 & 5.686 & 460.397 & 4 & 46.61 & 0 \\
\hline $5 \mathrm{l}$ & 4.583 & 5.752 & 5.752 & 480.815 & 4 & 46.61 & 0 \\
\hline $5 \mathrm{~m}$ & 4.583 & 5.747 & 5.747 & 480.815 & 4 & 46.61 & 0 \\
\hline $5 \mathrm{n}$ & 4.583 & 5.824 & 5.824 & 480.815 & 4 & 46.61 & 0 \\
\hline 50 & 4.477 & 5.458 & 5.458 & 464.361 & 4 & 46.61 & 0 \\
\hline
\end{tabular}

$\mathrm{S}+\log \mathrm{P}$ and MlogP are octanol-water distribution coefficients (It should be <5.0).

$\mathrm{S}+\log \mathrm{D}$ is $\mathrm{pH}$ dependent octanol-water distribution coefficient.

$\mathrm{HBDH}$ indicates number of hydrogen bond donors (It should be $<5 \mathrm{H}$-bond donors)

MNO value indicates total number of hydrogen bond acceptor (sum of $\mathrm{N} \& \mathrm{O}$ atoms). It should be $<10 \mathrm{H}$-bond acceptors.

Mol Wt is molecular weight (It should be 180-480 Daltons).

TPSA is the topological polar surface area expressed in square angstroms (It should be $<140 \AA^{2}$ ).

\section{Experimental}

\section{General experimental procedures}

All the chemicals and solvents were of analytical grade purchased from local supplier of Sigma Aldrich and Alfa Aesar. Melting points were measured by Gallen Kamp electrothermal apparatus. The purity of synthesized compounds was confirmed by using silica coated TLC plates $\mathrm{F}_{256} 20 \times 20 \mathrm{~cm}$. ${ }^{1} \mathrm{H}$ NMR spectra were recorded on $500 \mathrm{MHz}$ Bruker spectrometers while ${ }^{13} \mathrm{C}$ NMR spectra were taken at $125 \mathrm{MHz}$ using the same instrument. The chemical shift value $\delta$ was taken on ppm scale and TMS was used as internal reference standard. Jasco-320-A spectrophotometer was used to record IR spectra as $\mathrm{KBr}$ pellets. EI-MS and HR-EI-MS spectra were recorded on JMS-HX-110 spectrometer.

\section{Synthesis of sulfonamide (3)}

4-Bromobenzenesulfonyl chloride $(1 ; 0.02 \mathrm{~mol} ; 6 \mathrm{~g})$ was added with p-ethoxy aniline $(2 ; 0.02 \mathrm{~mol} ; 3 \mathrm{~mL})$ in $250 \mathrm{~mL}$ round bottom flask together with $50 \mathrm{~mL}$ water. The $\mathrm{pH}$ of the reaction mixture was adjusted at 10.0 by adding aqueous solution of $\mathrm{Na}_{2} \mathrm{CO}_{3}$ at room temperature. The reaction mixture was stirred continuously, and completion of the reaction was monitored by TLC. On completion of reaction, concentrated $\mathrm{HCl}$ was added drop wise to the mixture to adjust the $\mathrm{pH}$ to 2.0 to precipitate the product. The precipitates were filtered, washed with cold distilled water, and were crystallized in ethanol to get the off-white crystals of 4-bromo-N-(4-ethoxyphenyl) benzene sulfonamide (3) with $87 \%$ yield. Yield $87 \%$; Off white crystals; mp: $118-119^{\circ} \mathrm{C}$; IR $\left(\mathrm{KBr}, \mathrm{cm}^{-1}\right)$ $v_{\max }: 3322(\mathrm{~N}-\mathrm{H}), 3037$ (Ar-H), $2940(\mathrm{C}-\mathrm{H}), 1629-1568$ (Ar C=C), 1376 (S=0), 1237 (C-O). ${ }^{1} \mathrm{H}-\mathrm{NMR}\left(500 \mathrm{MHz}, \mathrm{CDCl}_{3}\right.$ ) $\delta(\mathrm{ppm}): 1.40$ $\left(\mathrm{t}, \mathrm{J}=6.5 \mathrm{~Hz}, 3 \mathrm{H}, \mathrm{CH}_{3}-\mathrm{CH}_{2}-\mathrm{O}\right), 3.98\left(\mathrm{q}, \mathrm{J}=6.5 \mathrm{~Hz}, 2 \mathrm{H}, \mathrm{CH}_{3}-\mathrm{CH}_{2}-\mathrm{O}\right.$ ), $6.78(\mathrm{~d}, \mathrm{~J}=8.5 \mathrm{~Hz}, 2 \mathrm{H}, \mathrm{H}-2,6), 6.87$ (d, J = $8.5 \mathrm{~Hz}, 2 \mathrm{H}, \mathrm{H}-3,5), 7.43$ (d, J = 8.5 Hz, 2H, H-2', $6^{\prime}$ ), 7.57 (d, J = $\left.8.5 \mathrm{~Hz}, 2 \mathrm{H}, \mathrm{H}-3^{\prime}, 5^{\prime}\right) .{ }^{13} \mathrm{C}-\mathrm{NMR}$ (125 MHz, $\left.\mathrm{CDCl}_{3}\right) \delta(\mathrm{ppm}): 14.8\left(\mathrm{CH}_{3}\right), 63.7\left(\mathrm{CH}_{2}\right), 114.7(\mathrm{C}-3,5)$, 127.9 (C-4'), 129.2 (C-2,6), 130.1 (C-2',6'), 130.6 (C-1), 132.3 (C3',5'), 137.6 (C-1'), 158.6 (C-4). HR-EI-MS (m/z) [M] ${ }^{+}: 354.9889$, calculated for $\mathrm{C}_{14} \mathrm{H}_{14} \mathrm{BrNO}_{3} \mathrm{~S} ; 354.9877$.

\section{Synthesis of $\mathrm{N}$-alkyl/aralkyl substituted sulphonamides (5a-0)}

The calculated amount of $3(0.1 \mathrm{mmol})$ was taken in $50 \mathrm{~mL}$ round bottomed flask and $10.0 \mathrm{~mL}$ of $\mathrm{N}, \mathrm{N}$-dimethyl formamide (DMF) was added followed by the addition of sodium hydride $(0.01 \mathrm{mmol})$. The mixture was stirred for 30 minutes at room temperature with onward addition of electrophiles alkyl/aralkyl halides (4a-o) (Table 1) separately to the reaction mixture 
which was further stirred for three hours. The reaction progress was monitored by TLC till the appearance of single spot on chromatogram. The targeted products (5a-o) were precipitated by adding cold water followed by filtration, washing with cold water and crystallization in methanol.

\section{Spectral characterization of the compounds 5a-o}

4-Bromo-N-(4-ethoxyphenyl)-N-ethylbenzenesulfonamide (5a)

Yield 63\%; mp: $118-119^{\circ} \mathrm{C}$; IR (KBr) $v_{\max }: 3039,2968,1625-$ 1576, 1351, $1258 \mathrm{~cm}^{-1} .{ }^{1} \mathrm{H}$ NMR (500 MHz, $\mathrm{CDCl}_{3}$ ) $\delta(\mathrm{ppm}): 1.05(\mathrm{t}$, $\left.\mathrm{J}=7.0 \mathrm{~Hz}, 3 \mathrm{H}, \mathrm{H}-2^{\prime \prime}\right), 1.39\left(\mathrm{t}, \mathrm{J}=7.0 \mathrm{~Hz}, 3 \mathrm{H}, \mathrm{CH}_{3}-\mathrm{CH}_{2}-0\right), 3.53$ (q, J = $7.0 \mathrm{~Hz}, 2 \mathrm{H}, \mathrm{H}-1^{\prime \prime}$ ), 3.99 (q, J = 7.0 Hz, 2H, $\mathrm{CH}_{3}-\mathrm{CH}_{2}-\mathrm{O}$ ), 6.79 (d, J = 8.5 $\mathrm{Hz}, 2 \mathrm{H}, \mathrm{H}-2,6), 6.89$ (d, J = $8.5 \mathrm{~Hz}, 2 \mathrm{H}, \mathrm{H}-3,5), 7.44$ (d, J = $8.5 \mathrm{~Hz}, 2 \mathrm{H}$, $\left.\mathrm{H}-2^{\prime}, 6^{\prime}\right), 7.56$ (d, J = $\left.8.5 \mathrm{~Hz}, 2 \mathrm{H}, \mathrm{H}-3^{\prime}, 5^{\prime}\right) .{ }^{13} \mathrm{C} \mathrm{NMR}\left(125 \mathrm{MHz}, \mathrm{CDCl}_{3}\right.$ ) $\delta(\mathrm{ppm}): 14.0\left(\mathrm{C}-2^{\prime \prime}\right), 14.8\left(\mathrm{CH}_{3}\right), 45.9\left(\mathrm{C}-1^{\prime \prime}\right), 63.7\left(\mathrm{CH}_{2}\right), 114.8(\mathrm{C}-$ 3,5), 127.4 (C-4'), 129.2 (C-2,6), 130.1 (C-2',6'), 130.6 (C-1), 132.0 (C-3',5'), 137.6 (C-1'), 158.6 (C-4). HR-EI-MS (m/z): 383.0199 [M] ${ }^{+}$ calculated for $\mathrm{C}_{16} \mathrm{H}_{18} \mathrm{BrNO}_{3} \mathrm{~S} ; 383.0190$.

\section{4-Bromo-N-(4-ethoxyphenyl)-N-propylbenzenesulfonamide} (5b)

Yield 70\%; mp: $118-120^{\circ} \mathrm{C}$; IR (KBr) $v_{\max }: 3035,2960,1620-$ 1587, 1355, $1250 \mathrm{~cm}^{-1} .{ }^{1} \mathrm{H}$ NMR (500 MHz, $\left.\mathrm{CDCl}_{3}\right) \delta(\mathrm{ppm}): 0.87(\mathrm{t}$, $\left.\mathrm{J}=6.5 \mathrm{~Hz}, 3 \mathrm{H}, \mathrm{H}-3^{\prime \prime}\right), 1.39\left(\mathrm{t}, \mathrm{J}=7.0 \mathrm{~Hz}, 3 \mathrm{H}, \mathrm{CH}_{3}-\mathrm{CH}_{2}-\mathrm{O}\right), 1.43(\mathrm{~m}$, $\left.2 \mathrm{H}, \mathrm{H}-2^{\prime \prime}\right), 3.42\left(\mathrm{t}, \mathrm{J}=7.0 \mathrm{~Hz}, 2 \mathrm{H}, \mathrm{H}-1^{\prime \prime}\right), 4.00\left(\mathrm{q}, \mathrm{J}=7.0 \mathrm{~Hz}, 2 \mathrm{H}, \mathrm{CH}_{3}\right.$ $\mathrm{CH}_{2}-0$ ) , 6.77 (d, J = 8.0 Hz, 2H, H-2,6), 6.90 (d, J = 8.0 Hz, 2H, H-3,5), 7.42 (d, J = $\left.8.5 \mathrm{~Hz}, 2 \mathrm{H}, \mathrm{H}-2^{\prime}, 6^{\prime}\right), 7.56$ (d, J = $8.5 \mathrm{~Hz}, 2 \mathrm{H}, \mathrm{H}-3^{\prime}, 5^{\prime}$ ). ${ }^{13} \mathrm{C}$ NMR (125 MHz, $\mathrm{CDCl}_{3}$ ) $\delta(\mathrm{ppm}): 10.9\left(\mathrm{C}-3^{\prime \prime}\right), 14.8\left(\mathrm{CH}_{3}\right), 21.4$ $\left(\mathrm{C}-2^{\prime \prime}\right), 52.5\left(\mathrm{C}-1^{\prime \prime}\right), 63.6\left(\mathrm{CH}_{2}\right), 114.8(\mathrm{C}-3,5), 127.4\left(\mathrm{C}-4^{\prime}\right), 129.2$ (C-2,6), 130.0 (C-2',6'), 130.9 (C-1), 132.0 (C-3',5'), 137.5 (C1'), 158.6 (C-4). HR-EI-MS ( $\mathrm{m} / \mathrm{z}$ ): 397.0361 [M] $^{+}$calculated for $\mathrm{C}_{17} \mathrm{H}_{20} \mathrm{BrNO}_{3} \mathrm{~S} ; 397.0347$.

\section{4 - B r o m o - N - ( 4 - e t hox y p h e n y l) - N - isopropylbenzenesulfonamide $(5 \mathrm{c})$}

Yield 69\%; mp: $118-119^{\circ} \mathrm{C}$; IR (KBr) $v_{\max }: 3037,2962,1618-$ 1595, 1357, $1255 \mathrm{~cm}^{-1} .{ }^{1} \mathrm{H}$ NMR (500 MHz, $\mathrm{CDCl}_{3}$ ) $\delta$ (ppm): 1.01 (d, J = $\left.6.5 \mathrm{~Hz}, 6 \mathrm{H}, \mathrm{H}-1^{\prime \prime}, 3^{\prime \prime}\right), 1.39$ (t, J = $\left.6.5 \mathrm{~Hz}, 3 \mathrm{H}, \mathrm{CH}_{3}-\mathrm{CH}_{2}-\mathrm{O}\right)$, 4.00 (q, J = $6.5 \mathrm{~Hz}, 2 \mathrm{H}, \mathrm{CH}_{3}-\mathrm{CH}_{2}-0$ ), 4.57 (m, ${ }^{1} \mathrm{H}, \mathrm{H}-1^{\prime \prime}$ ), 6.79 (d, $\mathrm{J}=8.5 \mathrm{~Hz}, 2 \mathrm{H}, \mathrm{H}-2,6), 6.88(\mathrm{~d}, \mathrm{~J}=8.5 \mathrm{~Hz}, 2 \mathrm{H}, \mathrm{H}-3,5), 7.56$ (br s, $\left.4 \mathrm{H}, \mathrm{H}-2^{\prime}, 3^{\prime}, 5^{\prime}, 6^{\prime}\right) .{ }^{13} \mathrm{C}$ NMR (125 MHz, $\left.\mathrm{CDCl}_{3}\right) \delta(\mathrm{ppm}): 14.8\left(\mathrm{CH}_{3}\right)$, $22.1\left(\mathrm{C}-1^{\prime \prime}, 3^{\prime \prime}\right), 51.3$ (C-2'), $63.7\left(\mathrm{CH}_{2}\right), 114.6(\mathrm{C}-3,5), 126.6\left(\mathrm{C}-4^{\prime}\right)$,

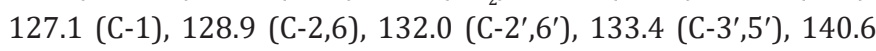
(C-1'), 159.1 (C-4). HR-EI-MS ( $\mathrm{m} / \mathrm{z}$ ): $397.0361[\mathrm{M}]^{+}$calculated for $\mathrm{C}_{17} \mathrm{H}_{20} \mathrm{BrNO}_{3} \mathrm{~S} ; 397.0347$.

\section{4 - B rom o - N - ( n - b u t yl) - N - ( 4 - e thox y p e n yl) benzenesulfonamide ( $5 \mathrm{~d})$}

Yield 60\%; mp: $124-125^{\circ} \mathrm{C}$; IR (KBr) $v_{\max }: 3035,2960,1617-$ 1596, 1357, $1255 \mathrm{~cm}^{-1} .{ }^{1} \mathrm{H}$ NMR (500 MHz, $\mathrm{CDCl}_{3}$ ) $\delta(\mathrm{ppm}): 0.84(\mathrm{t}$,
$\left.\mathrm{J}=7.0 \mathrm{~Hz}, 3 \mathrm{H}, \mathrm{H}-4^{\prime \prime}\right), 1.29$ (m, 2H, H-3") 1.36 (m, 2H, H-2"), 1.39 $\left(\mathrm{t}, \mathrm{J}=7.0 \mathrm{~Hz}, 3 \mathrm{H}, \mathrm{CH}_{3}-\mathrm{CH}_{2}-0\right), 3.45$ (t, J = 7.0 Hz, 2H, H-1"), 3.99 (q, $\left.\mathrm{J}=7.0 \mathrm{~Hz}, 2 \mathrm{H}, \mathrm{CH}_{3}-\mathrm{CH}_{2}-\mathrm{O}\right), 6.78(\mathrm{~d}, \mathrm{~J}=8.5 \mathrm{~Hz}, 2 \mathrm{H}, \mathrm{H}-3,5), 6.88(\mathrm{~d}$, $\mathrm{J}=8.5 \mathrm{~Hz}, 2 \mathrm{H}, \mathrm{H}-2,6), 7.42\left(\mathrm{~d}, \mathrm{~J}=8.5 \mathrm{~Hz}, 2 \mathrm{H}, \mathrm{H}-2^{\prime}, 6^{\prime}\right), 7.56(\mathrm{~d}, \mathrm{~J}=$ $\left.8.5 \mathrm{~Hz}, 2 \mathrm{H}, \mathrm{H}-3^{\prime}, 5^{\prime}\right) .{ }^{13} \mathrm{C}$ NMR (125 MHz, CDCl $) \delta(\mathrm{ppm}): 13.6$ (C$\left.4^{\prime \prime}\right), 14.8\left(\mathrm{CH}_{3}\right), 19.6\left(\mathrm{C}-3^{\prime \prime}\right), 30.2\left(\mathrm{C}-2^{\prime \prime}\right), 50.6\left(\mathrm{C}-1^{\prime \prime}\right), 63.7\left(\mathrm{CH}_{2}\right)$, 114.8 (C-3,5), 127.4 (C-4'), 129.2 (C-2,6), 129.9 (C-2',6'), 130.9 (C-1), 132.0 (C-3',5'), 137.4 (C-1'), 158.6 (C-4). HR-EI-MS (m/z): $411.0517[\mathrm{M}]^{+}$calculated for $\mathrm{C}_{18} \mathrm{H}_{22} \mathrm{BrNO}_{3} \mathrm{~S} ; 413.0503$.

\section{4 - B r o m o - N - ( s e c - b u t yl) - N - ( 4 - e thox y phe nyl) benzenesulfonamide $(5 e)$}

Yield 65\%; mp: $120-124^{\circ} \mathrm{C}$; IR (KBr) $v_{\max }: 3039,2969,1617-$ 1599, 1350, $1261 \mathrm{~cm}^{-1} .{ }^{1} \mathrm{H}$ NMR (500 MHz, $\mathrm{CDCl}_{3}$ ) $\delta(\mathrm{ppm}): 0.92(\mathrm{t}$, $\left.\mathrm{J}=7.0 \mathrm{~Hz}, 3 \mathrm{H}, \mathrm{H}-4^{\prime \prime}\right), 0.99$ (d, J = $\left.6.5 \mathrm{~Hz}, 3 \mathrm{H}, \mathrm{H}-1^{\prime \prime}\right), 1.20,1.34(\mathrm{~m}$, $\left.2 \mathrm{H}, \mathrm{H}-3^{\prime \prime}\right), 1.39$ (t, J = 7.0 Hz, 3H, $\left.\mathrm{CH}_{3}-\mathrm{CH}_{2}-\mathrm{O}\right), 4.00$ (q, J = $7.0 \mathrm{~Hz}$, $\left.2 \mathrm{H}, \mathrm{CH}_{3}-\mathrm{CH}_{2}-\mathrm{O}\right), 4.25\left(\mathrm{~m},{ }^{1} \mathrm{H}, \mathrm{H}-2^{\prime \prime}\right), 6.79$ (d, J = 8.5 Hz, 2H, H-2,6), $6.88(\mathrm{~d}, \mathrm{~J}=8.5 \mathrm{~Hz}, 2 \mathrm{H}, \mathrm{H}-3,5), 7.54(\mathrm{~m}, 4 \mathrm{H}, \mathrm{H}-2,3,5,6) .{ }^{13} \mathrm{C} \mathrm{NMR}$ (125 MHz, $\left.\mathrm{CDCl}_{3}\right) \delta(\mathrm{ppm}): 11.5\left(\mathrm{C}-4^{\prime \prime}\right), 14.8\left(\mathrm{CH}_{3}\right), 19.9\left(\mathrm{C}-1^{\prime \prime}\right)$, $28.6\left(\mathrm{C}-3^{\prime \prime}\right), 57.5\left(\mathrm{C}-2^{\prime \prime}\right), 63.6\left(\mathrm{CH}_{2}\right), 114.5(\mathrm{C}-3,5), 126.8\left(\mathrm{C}-4^{\prime}\right)$, 127.0 (C-1), 128.9 (C-2,6), 132.0 (C-2',6'), 133.3 (C-3',5'), 140.6 (C-1'), 159.1 (C-4). HR-EI-MS (m/z): 411.0517 [M] calculated for $\mathrm{C}_{18} \mathrm{H}_{22} \mathrm{BrNO}_{3} \mathrm{~S} ; 413.0503$.

\section{4-Bromo-N-(4-ethoxyphenyl)-N-pentylbenzenesulfonamide} (5f)

Yield 68\%; mp: $120-122^{\circ} \mathrm{C}$; IR (KBr) $v_{\max }: 3055,2965,1619-$ 1597, 1351, $1260 \mathrm{~cm}^{-1} .{ }^{1} \mathrm{H}$ NMR (500 MHz, $\left.\mathrm{CDCl}_{3}\right) \delta(\mathrm{ppm}): 0.82$ $\left(\mathrm{t}, \mathrm{J}=7.0 \mathrm{~Hz}, 3 \mathrm{H}, \mathrm{H}-5^{\prime \prime}\right), 1.21-1.36\left(\mathrm{~m}, 6 \mathrm{H}, \mathrm{H}-2^{\prime \prime}-4^{\prime \prime}\right), 1.39$ (t, J = 7.0 $\mathrm{Hz}, 3 \mathrm{H}, \mathrm{CH}_{3}-\mathrm{CH}_{2}-\mathrm{O}$ ), $3.44\left(\mathrm{t}, \mathrm{J}=7.0 \mathrm{~Hz}, 2 \mathrm{H}, \mathrm{H}-1^{\prime \prime}\right), 3.99$ (q, J = 7.0 $\mathrm{Hz}, 2 \mathrm{H}, \mathrm{CH}_{3}-\mathrm{CH}_{2}-\mathrm{O}$ ), 6.78 (d, J = 8.5 Hz, 2H, H-2,6), 6.88 (d, J = 8.5 $\mathrm{Hz}, 2 \mathrm{H}, \mathrm{H}-3,5$ ), 7.42 (d, J = 8.5 Hz, 2H, H-2',6'), 7.55 (d, J = $8.5 \mathrm{~Hz}$, 2H, H-3',5'). ${ }^{13} \mathrm{C}$ NMR (125 MHz, $\mathrm{CDCl}_{3}$ ) $\delta$ (ppm): 14.0 (C-5"), 14.8 $\left(\mathrm{CH}_{3}\right), 22.2\left(\mathrm{C}-4^{\prime \prime}\right), 27.8\left(\mathrm{C}-3^{\prime \prime}\right), 28.5\left(\mathrm{C}-2^{\prime \prime}\right), 50.8\left(\mathrm{C}-1^{\prime \prime}\right), 63.7\left(\mathrm{CH}_{2}\right)$, 114.7 (C-2,5), 127.3 (C-4'), 129.2 (C-2,6), 129.9 (C-2',6'), 130.9 (C-1), $132.0\left(\mathrm{C}-3^{\prime}, 5^{\prime}\right), 137.5$ (C-1'), 158.6 (C-4). HR-EI-MS (m/z): $425.0675[\mathrm{M}]^{+}$calculated for $\mathrm{C}_{19} \mathrm{H}_{24} \mathrm{BrClNO}_{3} \mathrm{~S} ; 425.0660$.

\section{4-Bromo-N-(4-ethoxyphenyl)-N-heptylbenzenesulfonamide} (5g)

Yield 66\%; mp: $120-123^{\circ} \mathrm{C}$; IR (KBr) $v_{\max }: 3051,2962,1621-$ 1595, 1353, $1252 \mathrm{~cm}^{-1} .{ }^{1} \mathrm{H}$ NMR (500 MHz, $\mathrm{CDCl}_{3}$ ) $\delta$ (ppm): 0.82 ( $\left.\mathrm{t}, \mathrm{J}=6.8 \mathrm{~Hz}, 3 \mathrm{H}, \mathrm{H}-\mathrm{7}^{\prime \prime}\right), 1.18-1.36\left(\mathrm{~m}, 10 \mathrm{H}, \mathrm{H}-2^{\prime \prime}-6^{\prime \prime}\right), 1.39$ (t, J = $\left.7.0 \mathrm{~Hz}, 3 \mathrm{H}, \mathrm{CH}_{3}-\mathrm{CH}_{2}-0\right), 3.44\left(\mathrm{t}, \mathrm{J}=6.8 \mathrm{~Hz}, 2 \mathrm{H}, \mathrm{H}-1^{\prime \prime}\right), 3.98$ (q, J = $\left.7.0 \mathrm{~Hz}, 2 \mathrm{H}, \mathrm{CH}_{3}-\mathrm{CH}_{2}-\mathrm{O}\right), 6.76$ (d, J = 8.4 Hz, 2H, H-2,6), 6.88 (d, J $=8.4 \mathrm{~Hz}, 2 \mathrm{H}, \mathrm{H}-3,5), 7.41\left(\mathrm{~d}, \mathrm{~J}=8.4 \mathrm{~Hz}, 2 \mathrm{H}, \mathrm{H}-2^{\prime}, 6^{\prime}\right), 7.55(\mathrm{~d}, \mathrm{~J}=$ $\left.8.4 \mathrm{~Hz}, 2 \mathrm{H}, \mathrm{H}-3^{\prime}, 5^{\prime}\right) .{ }^{13} \mathrm{C}$ NMR $\left(125 \mathrm{MHz}, \mathrm{CDCl}_{3}\right) \delta(\mathrm{ppm}): 14.1$ (C7"), $14.8\left(\mathrm{CH}_{3}\right), 22.6$ (C-6"), $26.3\left(\mathrm{C}-5^{\prime \prime}\right), 28.2\left(\mathrm{C}-4^{\prime \prime}\right), 28.8\left(\mathrm{C}-3^{\prime \prime}\right)$, $50.9\left(\mathrm{C}-2^{\prime \prime}\right), 63.7\left(\mathrm{CH}_{2}\right), 114.7(\mathrm{C}-3,5), 127.4\left(\mathrm{C}-4^{\prime}\right), 129.2(\mathrm{C}-2,6)$, 129.9 (C-2',6'), 130.9 (C-1'), 132.0 (C-3',5'), 137.5 (C-1'), 158.6 (C4). HR-EI-MS ( $\mathrm{m} / \mathrm{z}): 453.0987[\mathrm{M}]^{+}$calculated for $\mathrm{C}_{21} \mathrm{H}_{28} \mathrm{BrClNO}_{3} \mathrm{~S}$; 453.0976 . 
4-Bromo-N-(4-ethoxyphenyl)-N-octylbenzenesulfonamide (5h)

Yield 71\%; mp: $121-123^{\circ} \mathrm{C}$; IR (KBr) $v_{\max }: 3052,2960,1617-$ 1599, 1350, $1252 \mathrm{~cm}^{-1} .{ }^{1} \mathrm{H}$ NMR (500 MHz, $\mathrm{CDCl}_{3}$ ) $\delta$ (ppm): 0.84 ( $\left.\mathrm{t}, \mathrm{J}=7.0 \mathrm{~Hz}, 3 \mathrm{H}, \mathrm{H}-8^{\prime \prime}\right), 1.18-1.36\left(\mathrm{~m}, 12 \mathrm{H}, \mathrm{H}-2^{\prime \prime}-7^{\prime \prime}\right), 1.40$ (t, J = $\left.7.0 \mathrm{~Hz}, 3 \mathrm{H}, \mathrm{CH}_{3}-\mathrm{CH}_{2}-\mathrm{O}\right), 3.44\left(\mathrm{t}, \mathrm{J}=7.0 \mathrm{~Hz}, 2 \mathrm{H}, \mathrm{H}-1^{\prime \prime}\right), 3.99$ (q, J = $\left.7.0 \mathrm{~Hz}, 2 \mathrm{H}, \mathrm{CH}_{3}-\mathrm{CH}_{2}-0\right), 6.78(\mathrm{~d}, \mathrm{~J}=7.5 \mathrm{~Hz}, 2 \mathrm{H}, \mathrm{H}-2,6), 6.88$ (d, J $=7.5 \mathrm{~Hz}, 2 \mathrm{H}, \mathrm{H}-3,5), 7.42\left(\mathrm{~d}, \mathrm{~J}=7.5 \mathrm{~Hz}, 2 \mathrm{H}, \mathrm{H}-2^{\prime}, 6^{\prime}\right), 7.56(\mathrm{~d}, \mathrm{~J}=$ $\left.7.5 \mathrm{~Hz}, 2 \mathrm{H}, \mathrm{H}-3^{\prime}, 5^{\prime}\right) .{ }^{13} \mathrm{C}$ NMR (125 MHz, $\mathrm{CDCl}_{3}$ ) $\delta(\mathrm{ppm}): 14.1$ (C$\left.8^{\prime \prime}\right), 14.8\left(\mathrm{CH}_{3}\right), 22.6\left(\mathrm{C}-7^{\prime \prime}\right), 31.7\left(\mathrm{C}-6^{\prime \prime}\right), 29.0\left(\mathrm{C}-5^{\prime \prime}\right), 28.1\left(\mathrm{C}-4^{\prime \prime}\right)$, 26.3 (C-3"), 29.1 (C-2"), 50.9 (C-1"), $63.7\left(\mathrm{CH}_{2}\right), 114.7(\mathrm{C}-3,5)$, $127.4\left(\mathrm{C}-4^{\prime}\right), 129.2(\mathrm{C}-2,6), 129.9\left(\mathrm{C}-2^{\prime}, 6^{\prime}\right), 130.9$ (C-1), 132.0 (C3',5'), 137.5 (C-1'), 158.5 (C-4). HR-EI-MS (m/z): 467.1144 [M] ${ }^{+}$ calculated for $\mathrm{C}_{22} \mathrm{H}_{30} \mathrm{BrNO}_{3} \mathrm{~S} ; 467.1132$.

Ethyl N-((4-bromophenyl) sulfonyl)-N-(4-ethoxyphenyl) glycinate (5i)

Yield 53\%; mp: $123-124^{\circ} \mathrm{C}$; IR (KBr) $v_{\max }: 3037,2962,1739$, 1618-1595, 1357, $1255 \mathrm{~cm}^{-1} .{ }^{1} \mathrm{H}$ NMR (500 MHz, $\mathrm{CDCl}_{3}$ ) $\delta(\mathrm{ppm})$ : $1.20\left(\mathrm{t}, \mathrm{J}=7.0 \mathrm{~Hz}, 3 \mathrm{H}, \mathrm{CH}_{3}-\mathrm{CH}_{2}-\mathrm{O}\right), 1.38\left(\mathrm{t}, \mathrm{J}=7.0 \mathrm{~Hz}, 3 \mathrm{H}, \mathrm{CH}_{3}-\right.$ $\mathrm{CH}_{2}-\mathrm{O}$ ), $3.97\left(\mathrm{q}, \mathrm{J}=7.0 \mathrm{~Hz}, 2 \mathrm{H}, \mathrm{CH}_{3}-\mathrm{CH}_{2}-\mathrm{O}\right), 4.13(\mathrm{q}, \mathrm{J}=7.0 \mathrm{~Hz}, 2 \mathrm{H}$, $\mathrm{CH}_{3}-\mathrm{CH}_{2}-\mathrm{O}$ ), 4.35 (s, 2H, N-CH $\left.{ }_{2}\right), 6.75$ (d, J = $\left.8.5 \mathrm{~Hz}, 2 \mathrm{H}, \mathrm{H}-2,6\right), 7.07$ (d, J = 8.5 Hz, 2H, H-3,5), 7.51 (d, J = 8.5 Hz, 2H, H-2',6'), 7.56 (d, J = $\left.8.5 \mathrm{~Hz}, 2 \mathrm{H}, \mathrm{H}-3^{\prime}, 5^{\prime}\right) .{ }^{13} \mathrm{C}$ NMR (125 MHz, $\left.\mathrm{CDCl}_{3}\right) \delta(\mathrm{ppm}): 14.1\left(\mathrm{CH}_{3}\right)$, $14.8\left(\mathrm{CH}_{3}\right), 53.2\left(\mathrm{C}-2^{\prime \prime}\right), 61.5\left(\mathrm{CH}_{2}\right), 63.7\left(\mathrm{CH}_{2}\right), 114.9(\mathrm{C}-3,5), 127.8$ (C-4'), 129.4 (C-2,6), 130.5 (C-2',6'), 131.6 (C-1), 131.9 (C-3',5'), 138.2 (C-1'), 159.0 (C-4), 168.9 (C=0). HR-EI-MS ( $\mathrm{m} / \mathrm{z}): 441.0261$ $[\mathrm{M}]^{+}$calculated for $\mathrm{C}_{18} \mathrm{H}_{20} \mathrm{BrNO}_{5} \mathrm{~S} ; 443.0245$.

\section{N-Benzyl-4-bromo-N-(4-ethoxyphenyl) benzenesulfonamide (5j)}

Yield 69\%; mp: $118-120^{\circ} \mathrm{C}$; IR (KBr) $v_{\text {max }}: 3039,2969,1617-$ 1599, 1350, $1261 \mathrm{~cm}^{-1} .{ }^{1} \mathrm{H}$ NMR (500 MHz, $\mathrm{CDCl}_{3}$ ) $\delta(\mathrm{ppm}): 1.35$ ( $\left.\mathrm{t}, \mathrm{J}=7.0 \mathrm{~Hz}, 3 \mathrm{H}, \mathrm{CH}_{3}-\mathrm{CH}_{2}-\mathrm{O}\right), 3.92\left(\mathrm{q}, \mathrm{J}=7.0 \mathrm{~Hz}, 2 \mathrm{H}, \mathrm{CH}_{3}-\mathrm{CH}_{2}-\mathrm{O}\right)$, 4.65 (s, 2H, H-7"), 6.68 (d, J = $8.5 \mathrm{~Hz}, 2 \mathrm{H}, \mathrm{H}-2,6), 6.81$ (d, J = 8.5 $\mathrm{Hz}, 2 \mathrm{H}, \mathrm{H}-3,5), 7.17-7.24$ (m, 5H, H-2"'-6"), 7.49 (d, J = 8.5 Hz, 2H, $\left.\mathrm{H}-2^{\prime}, 6^{\prime}\right), 7.60$ (d, J = $\left.8.5 \mathrm{~Hz}, 2 \mathrm{H}, \mathrm{H}-3^{\prime}, 5^{\prime}\right) .{ }^{13} \mathrm{C}$ NMR (125 MHz, $\mathrm{CDCl}_{3}$ ) $\delta$ (ppm): $14.8\left(\mathrm{CH}_{3}\right), 55.2\left(\mathrm{C}-7^{\prime \prime}\right), 63.6\left(\mathrm{CH}_{2}\right), 114.7(\mathrm{C}-3,5), 127.6$ (C-4') $127.7\left(\mathrm{C}-4^{\prime \prime}\right), 128.4\left(\mathrm{C}-3^{\prime \prime}, 5^{\prime \prime}\right), 128.7(\mathrm{C}-2,6), 129.2\left(\mathrm{C}-2^{\prime \prime}, 6^{\prime \prime}\right)$, 130.2 (C-2',6'), 130.8 (C-1), 132.2 (C-3',5'), 135.7 (C-1'), 137.8 (C$\left.1^{\prime}\right), 158.5$ (C-4). HR-EI-MS (m/z): 445.0359 [M] $^{+}$calculated for $\mathrm{C}_{21} \mathrm{H}_{19} \mathrm{BrClNO}_{3} \mathrm{~S} ; 445.0347$.

\section{4 - Bromo-N-(4-ethoxyphenyl)-N-(2 - methylbenzyl) benzenesulfonamide (5k)}

Yield $72 \%$; mp: $120-122^{\circ} \mathrm{C}$; IR (KBr) $v_{\text {max }}: 3039,2969,1617-$ 1599, 1350, $1261 \mathrm{~cm}^{-1} .{ }^{1} \mathrm{H}$ NMR (500 MHz, $\mathrm{CDCl}_{3}$ ) $\delta$ (ppm): 1.34 ( $\left.\mathrm{t}, \mathrm{J}=6.5 \mathrm{~Hz}, 3 \mathrm{H}, \mathrm{CH}_{3}-\mathrm{CH}_{2}-\mathrm{O}\right), 2.30\left(\mathrm{~s}, 3 \mathrm{H}, \mathrm{CH}_{3}\right), 3.91(\mathrm{q}, \mathrm{J}=6.5 \mathrm{~Hz}$, $2 \mathrm{H}, \mathrm{CH}_{3}-\mathrm{CH}_{2}-\mathrm{O}$ ), 4.67 (s, 2H, H-7"), 6.65 (d, J = $8.5 \mathrm{~Hz}, 2 \mathrm{H}, \mathrm{H}-2,6$ ), 6.76 (d, J = 8.5 Hz, 2H, H-3,5), 6.97-7.06 (m, 4H, H-3"'-6"), 7.50 (d, $\left.\mathrm{J}=8.0 \mathrm{~Hz}, 2 \mathrm{H}, \mathrm{H}-2^{\prime}, 6^{\prime}\right), 7.61\left(\mathrm{~d}, \mathrm{~J}=8.0 \mathrm{~Hz}, 2 \mathrm{H}, \mathrm{H}-3^{\prime}, 5^{\prime}\right) .{ }^{13} \mathrm{C} \mathrm{NMR}$ $\left(125 \mathrm{MHz}, \mathrm{CDCl}_{3}\right) \delta(\mathrm{ppm}): 14.7\left(\mathrm{CH}_{3}\right), 19.2\left(\mathrm{CH}_{3}\right), 52.9\left(\mathrm{C}-7^{\prime \prime}\right), 63.5$ $\left(\mathrm{CH}_{2}\right), 114.6$ (C-3,5), 125.8 (C-4") 127.7 (C-4'), 127.9 (C-5") 129.4
(C-2,6), 130.0 (C-2',6'), 130.2 (C-3"), 130.4 (C-6"), 130.6 (C-1), 132.1 (C-3',5'), 133.1 (C-1), 137.1 (C-1'), 137.4 (C-2'), 158.5 (C4). HR-EI-MS (m/z): $459.0515[\mathrm{M}]^{+}$calculated for $\mathrm{C}_{22} \mathrm{H}_{22} \mathrm{BrNO}_{3} \mathrm{~S}$; 459.0503 .

\section{4-Bromo-N-(2 - chlorobenzyl)-N-(4-ethoxyphenyl) benzenesulfonamide (5l)}

Yield 73\%; mp: $121-123^{\circ} \mathrm{C}$; IR (KBr) $v_{\max }: 3039,2969,1617-$ 1599, 1350, $1261 \mathrm{~cm}^{-1} .{ }^{1} \mathrm{H}$ NMR (500 MHz, $\left.\mathrm{CDCl}_{3}\right) \delta(\mathrm{ppm}): 1.35(\mathrm{t}$, $\left.\mathrm{J}=6.5 \mathrm{~Hz}, 3 \mathrm{H}, \mathrm{CH}_{3}-\mathrm{CH}_{2}-\mathrm{O}\right), 3.93\left(\mathrm{q}, \mathrm{J}=6.5 \mathrm{~Hz}, 2 \mathrm{H}, \mathrm{CH}_{3}-\mathrm{CH}_{2}-\mathrm{O}\right), 4.82$ (s, 2H, H-7"), 6.69 (d, J = $8.5 \mathrm{~Hz}, 2 \mathrm{H}, \mathrm{H}-2,6), 6.90$ (d, J = 8.5 Hz, 2H, H-3,5), 7.10-7.24 (m, 3H, H-4"'-6"'), 7.46 (d, J = 8.5 Hz, H-2', 6'), 7.52 (d, J = 8.0 Hz, $\left.{ }^{1} \mathrm{H}, \mathrm{H}-3^{\prime \prime}\right), 7.61$ (d, J = $\left.8.5 \mathrm{~Hz}, 2 \mathrm{H}, \mathrm{H}-3^{\prime}, 5^{\prime}\right) .{ }^{13} \mathrm{C}$ NMR (125 MHz, $\left.\mathrm{CDCl}_{3}\right) \delta(\mathrm{ppm}): 14.8\left(\mathrm{CH}_{3}\right), 52.1\left(\mathrm{C}-7^{\prime \prime}\right), 63.6\left(\mathrm{CH}_{2}\right)$, 114.7 (C-3,5), 127.0 (C-4"), 127.8 (C-4'), 128.9 (C-5"), 129.3 (C2,6), 129.4 (C-3"), 130.0 (C-2',6'), 130.5 (C-6"), 130.9 (C-1), 132.2 (C-3',5'), 133.4 (C-2), 133.5 (C-1"), 137.3 (C-1'), 158.6 (C-4). HR-EI-MS $(\mathrm{m} / \mathrm{z})$ : $478.9959[\mathrm{M}]^{+}$calculated for $\mathrm{C}_{21} \mathrm{H}_{19} \mathrm{BrClNO}_{3} \mathrm{~S}$; 478.9947.

\section{4 - Bromo-N-(3 - chlorobenzyl)-N-(4-ethoxyphenyl) benzenesulfonamide $(5 \mathrm{~m})$}

Yield 72\%; mp: $120-122^{\circ} \mathrm{C}$; IR (KBr) $v_{\max }: 3039,2969,1617-$ 1599, 1350, $1261 \mathrm{~cm}^{-1} .{ }^{1} \mathrm{H}$ NMR (500 MHz, $\mathrm{CDCl}_{3}$ ) $\delta(\mathrm{ppm}): 1.35$ $\left(\mathrm{t}, \mathrm{J}=7.0 \mathrm{~Hz}, 3 \mathrm{H}, \mathrm{CH}_{3}-\mathrm{CH}_{2}-\mathrm{O}\right), 3.94\left(\mathrm{q}, \mathrm{J}=7.0 \mathrm{~Hz}, 2 \mathrm{H}, \mathrm{CH}_{3}-\mathrm{CH}_{2}-\mathrm{O}\right)$, $4.62\left(\mathrm{~s}, 2 \mathrm{H}, \mathrm{H}-7^{\prime \prime}\right), 6.70(\mathrm{~d}, \mathrm{~J}=8.5 \mathrm{~Hz}, 2 \mathrm{H}, \mathrm{H}-2,6), 6.81$ (d, J = $8.5 \mathrm{~Hz}$, $2 \mathrm{H}, \mathrm{H}-3,5), 7.13-7.19$ (m, 4H, H-2", $4^{\prime \prime}-6^{\prime \prime}$ ), 7.48 (d, J = 8.5 Hz, 2H, $\left.\mathrm{H}-2^{\prime}, 6^{\prime}\right), 7.60\left(\mathrm{~d}, \mathrm{~J}=8.5 \mathrm{~Hz}, 2 \mathrm{H}, \mathrm{H}-3^{\prime}, 5^{\prime}\right) .{ }^{13} \mathrm{C} \mathrm{NMR}\left(125 \mathrm{MHz}, \mathrm{CDCl}_{3}\right)$ $\delta(\mathrm{ppm}): 14.8\left(\mathrm{CH}_{3}\right), 54.7\left(\mathrm{C}-7^{\prime \prime}\right), 63.6\left(\mathrm{CH}_{2}\right), 114.8(\mathrm{C}-3,5), 126.8$ (C-4"), 127.8 (C-4'), 128.0 (C-6"), $127.8\left(\mathrm{C}-2^{\prime \prime}\right), 128.6\left(\mathrm{C}-2^{\prime \prime}\right)$, $129.2(\mathrm{C}-2,6), 129.7\left(\mathrm{C}-5^{\prime \prime}\right), 130.1$ (C-2',6'), 130.6 (C-1), 132.2 (C3',5'), 134.3 (C-3"'), 137.4 (C-1'), 138.0 (C-1'), 158.7 (C-4). HR-EIMS (m/z): $478.9959[\mathrm{M}]^{+}$calculated for $\mathrm{C}_{21} \mathrm{H}_{19} \mathrm{BrClNO}_{3} \mathrm{~S} ; 478.9947$.

\section{4 - Bromo-N-(4-chlorobenzyl)- N-(4-ethoxyphenyl) benzenesulfonamide $(5 \mathrm{n})$}

Yield 70\%; mp: $120-122^{\circ} \mathrm{C}$; IR (KBr) $v_{\max }: 3039,2969,1617-$ 1599, 1350, $1261 \mathrm{~cm}^{-1} .{ }^{1} \mathrm{H}$ NMR (500 MHz, $\mathrm{CDCl}_{3}$ ) $\delta$ (ppm): 1.35 (t, J = 7.0 Hz, 3H, CH $\left.-\mathrm{CH}_{2}-0\right), 3.93\left(\mathrm{q}, \mathrm{J}=7.0 \mathrm{~Hz}, 2 \mathrm{H}, \mathrm{CH}_{3}-\mathrm{CH}_{2}-\mathrm{O}\right)$, 4.61 (s, 2H, H-7") 6.69 (d, J = 8.5 Hz, 2H, H-2,6), 6.78 (d, J = 8.5 $\mathrm{Hz}, 2 \mathrm{H}, \mathrm{H}-3,5), 7.12$ (d, J = 8.0 Hz, 2H, H-2", $\left.6^{\prime \prime}\right), 7.18$ (d, J = $8.0 \mathrm{~Hz}$, $2 \mathrm{H}, \mathrm{H}-3^{\prime \prime}, 5^{\prime \prime}$ ), 7.48 (d, J = $8.5 \mathrm{~Hz}, 2 \mathrm{H}, \mathrm{H}-2^{\prime}, 6^{\prime}$ ), 7.60 (d, J = $8.5 \mathrm{~Hz}$, $\left.2 \mathrm{H}, \mathrm{H}-3^{\prime}, 5^{\prime}\right) .{ }^{13} \mathrm{C}$ NMR (125 MHz, $\left.\mathrm{CDCl}_{3}\right) \delta(\mathrm{ppm}): 14.8\left(\mathrm{CH}_{3}\right), 54.5$ (C-7") $63.6\left(\mathrm{CH}_{2}\right), 114.8(\mathrm{C}-3,5), 127.8\left(\mathrm{C}-4^{\prime}\right), 128.7$ (C-2,6), 129.2 (C-2',6'), $130.0\left(\mathrm{C}-2^{\prime \prime}, 6^{\prime \prime}\right), 130.1\left(\mathrm{C}-3^{\prime \prime}, 5^{\prime \prime}\right), 130.5$ (C-1), 132.2 (C3',5'), 133.6 (C-4'), 134.4 (C-1"'), 137.6 (C-1'), 158.6 (C-4). HR-EIMS (m/z): $478.9959[\mathrm{M}]^{+}$calculated for $\mathrm{C}_{21} \mathrm{H}_{19} \mathrm{BrClNO}_{3} \mathrm{~S} ; 478.9947$.

\section{4 - Bromo- - - ( 4 - flourobenzyl) - N - (4 - ethoxyphenyl) benzenesulfonamide (5o)}

Yield 66\%; mp: $120-121^{\circ} \mathrm{C}$; IR (KBr) $v_{\text {max }}: 3039,2969,1617-$ 1599, 1350, $1261 \mathrm{~cm}^{-1} .{ }^{1} \mathrm{H}$ NMR (500 MHz, $\mathrm{CDCl}_{3}$ ) $\delta(\mathrm{ppm}): 1.35$ (t, J = 7.0 Hz, 3H, $\mathrm{CH}_{3}-\mathrm{CH}_{2}-\mathrm{O}$ ), $3.93\left(\mathrm{q}, \mathrm{J}=7.0 \mathrm{~Hz}, 2 \mathrm{H}, \mathrm{CH}_{3}-\mathrm{CH}_{2}-\mathrm{O}\right.$ ), 
4.61 (s, 2H, H-7") 6.69 (d, J = $8.5 \mathrm{~Hz}, 2 \mathrm{H}, \mathrm{H}-2,6), 6.78$ (d, J = $8.5 \mathrm{~Hz}$, $2 \mathrm{H}, \mathrm{H}-3,5), 6.89$ (d, J = 8.5 Hz, 2H, H-2", $\left.6^{\prime \prime}\right), 7.15$ (d, J = $8.5 \mathrm{~Hz}, 2 \mathrm{H}$, H-3",5"), 7.48 (d, J = $8.5 \mathrm{~Hz}, 2 \mathrm{H}, \mathrm{H}-2^{\prime}, 6^{\prime}$ ), 7.60 (d, J = $8.5 \mathrm{~Hz}, 2 \mathrm{H}$, H-3',5'). ${ }^{13} \mathrm{C}$ NMR (125 MHz, $\left.\mathrm{CDCl}_{3}\right) \delta(\mathrm{ppm}): 14.8\left(\mathrm{CH}_{3}\right), 54.5(\mathrm{C}-$ $\left.7^{\prime \prime}\right), 63.6\left(\mathrm{CH}_{2}\right), 114.7(\mathrm{C}-3,5), 115.3\left(\mathrm{C}-3^{\prime \prime}, 5^{\prime \prime}\right), 127.7$ (C-4'), 129.2 (C-2,6), 130.2 (C-2',6'), 130.4 (C-2"',6"), 130.5 (C-1), 131.6 (C-1"), 132.2 (C-3',5'), 137.7 (C-1'), 158.6 (C-4), 163.3 (C-4'). HR-EI-MS $(\mathrm{m} / \mathrm{z}): 463.0265[\mathrm{M}]^{+}$calculated for $\mathrm{C}_{21} \mathrm{H}_{19} \mathrm{BrFNO}_{3} \mathrm{~S} ; 463.0253$.

\section{Enzyme Inhibition Assays}

\section{Acetylcholinesterase inhibition assay}

AChE inhibition activity was performed by the reported method with some modifications [17]. Reaction mixture of $100 \mu \mathrm{L}$ contained $60 \mu \mathrm{L} 50 \mathrm{mM}$ phosphate buffer of pH 7.7, $10 \mu \mathrm{L}(0.5$ $\mathrm{mM} /$ well) test compound and $10 \mu \mathrm{L}(0.005$ unit/well) of electric eel enzyme (Sigma Inc). Contents were pre-incubated at $37^{\circ} \mathrm{C}$ for $10 \mathrm{~min}$ and pre-read at $405 \mathrm{~nm}$. The reaction was initiated by the addition of $10 \mu \mathrm{L}$ of $0.5 \mathrm{mM} /$ well substrate, acetylthiocholine iodide, followed by the addition of $10 \mu \mathrm{L}$ DTNB (0.5 mM/well). Incubation was continued for further 30 minutes and absorbance was measured using Synergy HTX (BioTek, USA) 96-well plate reader. Eserine $(0.5 \mathrm{mM} /$ well) was used as a positive control. The percent inhibition was calculated by the help of following equation. The active compounds were serially diluted and assayed against the enzyme. The data obtained was used to calculate $\mathrm{IC}_{50}$ values, that is, the concentration at which the enzyme activity is inhibited by $50 \%$.

$$
\text { Inhibition }(\%)=\frac{\text { Control-Test }}{\text { Control }} \times 100
$$

\section{$\alpha$-Glucosidase inhibition assay}

The aglucosidase inhibition assay was performed according to the reported method with some modification [15]. Total volume of the reaction mixture of $100 \mu \mathrm{L}$ contained $70 \mu \mathrm{L}$ of $50 \mathrm{mM}$ phosphate buffer with $\mathrm{pH} 6.8,10 \mu \mathrm{L}(0.5 \mathrm{mM})$ test compound, followed by the addition of $10 \mu \mathrm{L}$ (0.057 units) yeast enzyme (Sigma Inc.). The contents were mixed, pre-incubated for $10 \mathrm{~min}$ at $37^{\circ} \mathrm{C}$ and preread at $400 \mathrm{~nm}$. The reaction was initiated by the addition of $10 \mu \mathrm{L}$ of $0.5 \mathrm{mM}$ substrate (p-nitrophenyl glucopyranoside). After 30 min of incubation at $37^{\circ} \mathrm{C}$, absorbance was measured at $400 \mathrm{~nm}$ using Synergy HTX microplate reader. Acarbose was used as positive control. All experiments were carried out in triplicates. The percentage inhibition and IC $_{50}$ values were determined as mentioned above for AChE.

\section{Conclusion}

The targeted N-substituted-(4-bromophenyl)-4ethoxybenzenesulfonamides (5a-o) were synthesized in good yields and these molecules possessed broad range spectrum against AChE (5l, 5n, 5g, 5j, 5h; $\mathrm{IC}_{50}$ values $52.63 \pm 0.14,82.75 \pm$ $0.16,92.13 \pm 0.15,92.52 \pm 0.16,98.72 \pm 0.12 \mu \mathrm{M}$, respectively) and $\alpha$-glucosidase (5h, 5j, 5c, 5d , 5l; $\mathrm{IC}_{50}$ values $57.38 \pm 0.19,123.36 \pm$ $0.19,123.42 \pm 0.19,124.35 \pm 0.15,124.74 \pm 0.18 \mu \mathrm{M}$, respectively) . Therefore, these studies conclude that the newly synthesized molecules might serve as promising drug candidates for further structural optimizations and drug designing studies.

\section{Acknowledgement}

We are thankful to Alexander von Humboldt ( $\mathrm{AvH}$ ) Foundation, Germany for their financial support.

\section{References}

1. Perlovich G L, Strakhova N N, Kazachenko V P, Volkova T V, Tkacher V V, et al. (2008) Sulfonamides as a subject to study molecular interactions in crystals and solutions: Sublimation, solubility, solvation, distribution and crystal structure. Int J Pharm 349: 300-313.

2. Scozzafava A, Supuran C T (1998) Carbonic anhydrase inhibitors: Ureido and thioureido derivatives of aromatic sulfonamides possessing increased affinities for isozyme I. A Novel Route to 2,5-DisubstitutedL,3,4-Thiadiazoles via Thioureas, and their Interaction with Isozymes I, II and IV. J Enz Inhib 13: 103-123.

3. El Sayed N S, El Bendary R E, El Ashry S M, El Kerdawy M M (2011) Synthesis and antitumor activity of new sulfonamide derivatives of thiadiazolo[3,2-a]pyrimidines. Eur J Med Chem 46: 3714-3720.

4. Garcia Galan M J, Diaz Cruz M S, Bercelo D (2008) Identification and determination of metabolites and degradation products of sulfonamide antibiotics. Trend Anal Chem 27: 1008-1022.

5. Di Fiore A, Monti S M, Innocenti A, Winum J Y, De Simone G, et al. (2010) Carbonic anhydrase inhibitors: crystallographic and solution binding studies for the interaction of a boron-containing aromatic sulfamide with mammalian isoforms I-XV. Bioorg Med Chem Lett 20: 3601-3605.

6. Smaine F Z, Pacchiano F, Rami M, Barragan Montero V, Vullo D, et al. (2008) Carbonic anhydrase inhibitors: 2-substituted-1,3,4thiadiazole-5-sulfamides act as powerful and selective inhibitors of the mitochondrial isozymes VA and VB over the cytosolic and membraneassociated carbonic anhydrases I, II and IV. Bioorg Med Chem Lett 18: 6332-6335.

7. Sondhi S M, Johar M, Singhal N, Dastidar S G, Shukla R, et al. (2000) synthesis and anticancer, antiinflammatory,and analgesic activity evaluation of some sulfa drug and acridine derivatives. Monatsh. Chem./Chemical Monthly 131: 511-520.

8. Dow R L, Paight E S, Schneider S R, Hadcock J R, Hargrove D M, et al. (2004) Potent and selective, sulfamide-based human $\beta 3$-adrenergic receptor agonists. Bioorg Med Chem Lett 3235-3240.

9. Patel S D, Habeski W M, Cheng A C, de la Cruz E, Loh C, et al. (2009) Corrigendum to "Affinity labeling of the proteasome by a belactosin A derived inhibitor. Bioorg Med Chem Lett 19: 3339-3343.

10. Ezabadi I R, Camoutsis C, Zoumpoulakis P, Geronikaki A, Soković M, et al. (2008) Bioorg Med Chem 16: 1150-1161.

11. Chen Z, Xu W, Liu K, Yang S, Fan H, et al. (2010) synthesis and antiviral activity of 5-(4-chlorophenl)-1,3,4-thiadiazole sulfonamides. molecules 15: 9046-9056.

12. Pohanka M (2014) inhibitors of acetylcholinesterase and butyrylcholinesterase meet immunity. Int J Mol Sci 15: 9809-9825. 
13. Benalla W, Bellahcen S, Bnouham M (2010) Antidiabetic medicinal plants as a source of alpha glucosidase inhibitors. Cur Diab Rev 6: 247 254.

14. Özil M, Balaydın H T, Sentürk M (2019) Synthesis of 5-methyl-2,4dihydro-3H-1,2,4-triazole-3-one's aryl Schiff base derivatives and investigation of carbonic anhydrase and cholinesterase (AChE, BuChE) inhibitory properties. Bioorg Chem 86: 705-713.

15. Chapdelaine P, Tremblay R R, Dube J Y (1978) P-Nitrophenol-alpha-Dglucopyranoside as substrate for measurement of maltase activity in human semen. Clin Chem 24: 208-211.

16. Hameed A, Zehra S T, Abbas S, Nisa R U, Mahmood T, et al. (2016) One-pot synthesis of tetrazole-1,2,5,6-tetrahydronicotinonitriles and cholinesterase inhibition: Probing the plausible reaction mechanism via computational studies. Bioorg Chem 65: 38-47.
17. Ellman G L, Courtney K D, Andres V, Featherstone R M (1961) A new and rapid colorimetric determination of acetylcholinesterase activity. Biochem Pharmacol 7: 88-90.

18. Zakeri Milani P, Tajerzadeh H, Islambolchilar Z, Barzegar S, Valizadeh H (2006) The relation between molecular properties of drugs and their transport across the intestinal membrane. DARU: J Pharm Sci 14: 164171.

19. Lead I T, BioSolve IT, GmbH, Germany.

20. Chaudhry F, Naureen S, Huma R, Shaukat A, al Rashida M, et al. (2017) In search of new $\alpha$-glucosidase inhibitors: Imidazolylpyrazole derivatives. Bioorg Chem 71: 102-109.

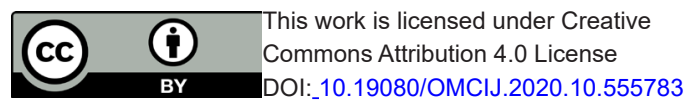

Your next submission with Juniper Publishers
will reach you the below assets
- Quality Editorial service
- Swift Peer Review
- Reprints availability
- E-prints Service
- Manuscript Podcast for convenient understanding
- Global attai nment for your research
- Manuscript accessibility in different formats
( Pdf, E-pub, Full Text, Audio)
- Unceasing customer service
Track the below URL for one-step submission
https://juniperpublishers.com/online-submission.php

